\title{
WEAKLY UNIFORM RANK TWO VECTOR BUNDLES ON MULTIPROJECTIVE SPACES
}

\author{
EDOARDO BALLICO and FRANCESCO MALASPINA ${ }^{凶}$
}

(Received 13 November 2010)

\begin{abstract}
Here we classify the weakly uniform rank two vector bundles on multiprojective spaces. Moreover, we show that every rank $r>2$ weakly uniform vector bundle with splitting type $a_{1,1}=\cdots=a_{r, s}=0$ is trivial and every rank $r>2$ uniform vector bundle with splitting type $a_{1}>\cdots>a_{r}$ splits.
\end{abstract}

2010 Mathematics subject classification: primary 14F05; secondary 14J60.

Keywords and phrases: uniform vector bundles, splitting type, multiprojective spaces.

\section{Introduction}

We denote by $\mathbb{P}^{n}$ the $n$-dimensional projective space over an algebraic field of characteristic zero. A rank $r$ vector bundle $E$ on $\mathbb{P}^{n}$ is said to be uniform if there is a sequence of integers $\left(a_{1}, \ldots, a_{r}\right)$ with $a_{1} \geq \cdots \geq a_{r}$ such that for every line $L$ on $\mathbb{P}^{n}, E_{\mid L} \cong \bigoplus_{i=1}^{r} \mathcal{O}\left(a_{i}\right)$. The sequence $\left(a_{1}, \ldots, a_{r}\right)$ is called the splitting type of $E$.

The classification of these bundles is known in many cases: rank $E \leq n$ for $n \geq 2$ (see [5, 9, 11]); rank $E=n+1$ for $n=2$ and $n=3$ (see [4, 6]); rank $E=5$ for $n=3$ (see [1]). Nevertheless, there are uniform vector bundles (of rank $2 n$ ) which are not homogeneous (see [3]).

In [2] the authors gave the notion of weakly uniform bundles on $\mathbb{P}^{1} \times \mathbb{P}^{1}$. For the study of rank two weakly uniform vector bundles on $\left(\mathbb{P}^{1}\right)^{s}$, see $[2,7,10]$.

Here we are interested in vector bundles on multiprojective spaces. Fix integers $s \geq 2$ and $n_{i} \geq 1$. Let $X:=\mathbb{P}^{n_{1}} \times \cdots \times \mathbb{P}^{n_{s}}$ be a multiprojective space. Let

$$
u_{i}: X \rightarrow \mathbb{P}^{n_{i}}
$$

be the projection on the $i$ th factor. For all $1<i<j$ let

$$
u_{i j}: X \rightarrow \mathbb{P}^{n_{i}} \times \mathbb{P}^{n_{j}}
$$

denote the projection onto the product of the $i$ th factor and the $j$ th factor. Set $\mathcal{O}:=\mathcal{O}_{X}$. For all integers $b_{1}, \ldots, b_{s}$ set $\mathcal{O}\left(b_{1}, \ldots, b_{s}\right):=\bigotimes_{i=1}^{s} u_{i}^{*}\left(\mathcal{O}_{\mathbb{P}^{n}}\left(b_{i}\right)\right)$. We recall that

(C) 2011 Australian Mathematical Publishing Association Inc. 0004-9727/2011 \$16.00 
every line bundle on $X$ is isomorphic to a unique line bundle $\mathcal{O}\left(b_{1}, \ldots, b_{s}\right)$. Set $X_{i}:=\prod_{j \neq i} \mathbb{P}^{n_{j}}$. Let

$$
\pi_{i}: X \rightarrow X_{i}
$$

be the projection. Hence, $\pi_{i}^{-1}(P) \cong \mathbb{P}^{n_{i}}$ for each $P \in X_{i}$. Let $E$ be a rank $r$ vector bundle on $X$. We say that $E$ is weakly uniform with splitting type $\left(a_{h, i}\right), 1 \leq h \leq r$, $1 \leq i \leq s$, if for all $i \in\{1, \ldots, s\}$, every $P \in X_{i}$ and every line $D \subseteq \pi_{i}^{-1}(P)$ the vector bundle $E \mid D$ on $D \cong \mathbb{P}^{1}$ has splitting type $a_{1, i} \geq \cdots \geq a_{r, i}$. A weakly uniform vector bundle $E$ on $X$ is called uniform if there is a line bundle $\left(a_{1}, \ldots, a_{S}\right)$ such that the splitting types of $E\left(a_{1}, \ldots, a_{s}\right)$ with respect to all $\pi_{i}$ are the same. In this case a splitting type of $E$ is the splitting type $c_{1} \geq \cdots \geq c_{r}, r:=\operatorname{rank}(E)$, of $E\left(a_{1}, \ldots, a_{s}\right)$. Notice that the $r$-tuple $\left(c_{1}, \ldots, c_{r}\right)$ of integers is not uniquely determined by $E$, but that the $(s-1)$-tuple $\left(c_{1}-c_{2}, \ldots, c_{s-1}-c_{s}\right)$ depends only on $E$. Indeed, a rank $r$ weakly uniform vector bundle $E$ of splitting type $\left(a_{h, i}\right), 1 \leq h \leq r, 1 \leq i \leq s$, is uniform if and only if there are $s-1$ integers $d_{j}, 2 \leq j \leq s$, such that $a_{h, i}=a_{h, 1}+d_{i}$ for all $i \in\{2, \ldots, s\}$. If $E$ is uniform, then the $r$-tuples $\left(a_{1,1}+y, \ldots, a_{r, 1}+y\right)$, $y \in \mathbb{Z}$, are exactly the splitting types of $E$. If $E$ is uniform, it is usually better to consider $E\left(0, a_{1,2}-a_{1,1}, \ldots, a_{1, s}-a_{1,1}\right)$ instead of $E$, because all the splitting types of $E\left(0, a_{1,2}-a_{1,1}, \ldots, a_{1, s}-a_{1,1}\right)$ as a weakly uniform vector bundle are the same.

In this paper we prove the following result.

THEOREM 1.1. Let $E$ be a rank two vector bundle on $X$. Then $E$ is weakly uniform if and only if there are $L \in \operatorname{Pic}(X)$, indices $1 \leq i<j \leq s$ and a rank two weakly uniform vector bundle $G$ on $\mathbb{P}^{n_{i}} \times \mathbb{P}^{n_{j}}$ such that $E \otimes L \cong u_{i j}^{*}(G)$. The vector bundle $E$ splits if either $n_{i} \geq 3$ or $n_{j} \geq 3$. If $1 \leq n_{1} \leq 2,1 \leq n_{2} \leq 2$ and $\left(n_{1}, n_{2}\right) \neq(1,1)$, then $E$ splits unless there is $h \in\{1,2\}$ such that $n_{h}=2$ and $E \otimes L \cong u_{h}^{*}\left(T \mathbb{P}^{2}\right)$ for some $L \in \operatorname{Pic}(X)$.

Moreover, we discuss the case of higher rank. We show that every rank $r>2$ weakly uniform vector bundle with splitting type $a_{1,1}=\cdots=a_{r, s}=0$ is trivial and every rank $r>2$ uniform vector bundle with splitting type $a_{1}>\cdots>a_{r}$ splits. Our methods do not allow us to attack other splitting types.

\section{Weakly uniform rank two vector bundles}

In order to prove Theorem 1.1 we need a few lemmas. We first consider the case $s=2$.

LEMMA 2.1. Assume $s=2, n_{1}=1$ and $n_{2}=2$. Let $E$ be a rank two vector bundle on $\mathbb{P}^{1} \times \mathbb{P}^{2}$. The vector bundle $E$ is weakly uniform if and only if either $E$ splits as the direct sum of two line bundles or there is a line bundle $L$ on $\mathbb{P}^{1} \times \mathbb{P}^{2}$ such that $E \cong L \otimes \pi_{2}^{*}\left(T \mathbb{P}^{2}\right)$.

ProOF. Since the 'if' part is obvious, it is sufficient to prove the 'only if' part. Let $\left(a_{h, i}\right), 1 \leq h \leq 2,1 \leq i \leq s$, be the splitting type of $E$. Up to a twist by a line 
bundle we may assume $a_{1,1}=a_{1,2}=0$. By rigidity or looking at the Chern classes $c_{i}\left(E \mid\{Q\} \times \mathbb{P}^{2}\right), i=1,2$, it is easy to see that if one of these two cases occurs for some $Q$, then it occurs for all $Q$. First assume $a_{2,2}=0$. Since the trivial line bundle on $\mathbb{P}^{1}$ is spanned, the theorem of changing basis implies that $F:=\pi_{2 *}(E)$ is a rank two vector bundle on $\mathbb{P}^{2}$ and that the natural map $\pi_{2}^{*}(F) \rightarrow E$ is an isomorphism [8, p. 11]. Since $E$ is weakly uniform, $F$ is uniform. The classification of all rank two uniform vector bundles on $\mathbb{P}^{2}$ shows that either $F$ splits or it is isomorphic to a twist of $T \mathbb{P}^{2}$ (see [5]), concluding the proof in the case $a_{2,2}=0$. Similarly, if $a_{2,1}=0$, there is a rank two vector bundle $G$ on $\mathbb{P}^{1}$ such that $\pi_{1}^{*}(G) \cong E$. Since every vector bundle on $\mathbb{P}^{1}$ splits, we have that $E$ splits also. Now we may assume $a_{2,2}<0$ and $a_{2,1}<0$. Since $a_{2,2}<0$, the base-change theorem gives that $\pi_{2 *}(E)$ is a line bundle, say of degree $b_{2}$, and that the natural map $\pi_{2}^{*} \pi_{2 *}(E) \rightarrow E$ has locally free cokernel [8, p. 11]. Thus, in this case $E$ fits in an exact sequence

$$
0 \rightarrow \mathcal{O}\left(0, b_{2}\right) \rightarrow E \rightarrow \mathcal{O}\left(a_{2,1},-b_{2}-a_{2,2}\right) \rightarrow 0 .
$$

The term $a_{2,1}$ in the last line bundle of (2.1) comes from $c_{1}(E)$. If (2.1) splits, then we are done. Since $a_{2,1} \leq 1$, Künneth's formula gives $H^{1}\left(\mathbb{P}^{1} \times \mathbb{P}^{2}, \mathcal{O}\left(-a_{2,1}, 2 b_{2}+\right.\right.$ $\left.\left.a_{2,2}\right)\right)=0$. Hence (2.1) splits.

LEMMA 2.2. Assume $s=2, n_{1}=1$ and $n_{2} \geq 3$. Then every rank two weakly uniform vector bundle on $X$ is the direct sum of two line bundles.

PROOF. We copy the proof of Lemma 2.1. Every rank two uniform vector bundle on $\mathbb{P}^{m}, m \geq 3$, splits. Hence $E$ splits even in the case $a_{2,2}=0$.

LEMMA 2.3. Assume $s=2$ and $n_{1}=n_{2}=2$. Let $E$ be a rank two indecomposable weakly uniform vector bundle on $X$. Then either $E \cong u_{1}^{*}\left(T \mathbb{P}^{2}\right)(u, v)$ or $E \cong$ $u_{2}^{*}\left(T \mathbb{P}^{2}\right)(u, v)$.

PROOF. Let $\left(a_{h, i}\right)$ be the splitting type of $E$. Up to a twist by a line bundle we may assume $a_{1,1}=a_{1,2}=0$. As in the proof of Lemma 2.1, the theorem of changing basis gives that either $E \cong u_{1}^{*}\left(T \mathbb{P}^{2}(-2)\right)$ or $E$ splits if $a_{2,1}=0$ and that $E \cong u_{2}^{*}\left(T \mathbb{P}^{2}(-2)\right)$ or $E$ splits if $a_{2,2}=0$. If $a_{2,1}<0$ and $a_{2,2}<0$, then we apply $\pi_{2 *}$ and get an exact sequence (2.1). Here Künneth's formula gives that (2.1) splits, without using any information on the integer $a_{2,2}$.

LEMMA 2.4. Assume $s=2, n_{1} \geq 3$ and $n_{2}=2$. Let $E$ be a rank two weakly uniform vector bundle on $X$. Then either $E$ splits or $E \cong u_{2}^{*}\left(T \mathbb{P}^{2}\right)(u, v)$ for some integers $u, v$.

PROOF. Let $\left(a_{h i}\right)$ be the splitting type of $E$. Up to a twist by a line bundle we may assume $a_{1,1}=a_{1,2}=0$. As in the proof of Lemma 2.1, the theorem of changing basis gives that $E \cong u_{1}^{*}\left(T \mathbb{P}^{2}(-2)\right)$ or $E$ splits if $a_{2,1}=0$ and that $E$ splits in the case $a_{1,2}<0$, because (2.1) splits by Künneth's formula.

LEMMA 2.5. Assume $s=2, n_{1} \geq 3$ and $n_{2} \geq 3$. Let $E$ be a rank two weakly uniform vector bundle on $X$. Then $E$ splits. 
Proof. Let $\left(a_{h i}\right)$ be the splitting type of $E$. Up to a twist by a line bundle we may assume $a_{1,1}=a_{1,2}=0$. If $a_{2,2}=0$, then base change gives $E \cong u_{2}^{*}(F)$ for some uniform vector bundle on $\mathbb{P}^{2}$. Thus, we may assume $a_{2,2}<0$. We have again the extension (2.1). Here again (2.1) splits by Künneth's formula.

Now we are ready to prove the main theorem.

Proof of TheOREM 1.1. First assume $s=2$. Theorem 1.1 says nothing in the case $n_{1}=n_{2}=1$ for which a full classification is not known ([2] shows that moduli arise). Lemmas 2.1, 2.2, 2.3, 2.4 and 2.5 cover all cases with $s=2$. Hence we may assume $s \geq 3$ and use induction on $s$. If $n_{i}=1$ for all $i$, then we may apply [2, Theorem 4]. For arbitrary $n_{i}$ the proof of [2, Theorem 4] works verbatim, but for the reader's sake we repeat that proof. Let $\left(a_{h i}\right)$ be the splitting type of $E$. Up to a twist by a line bundle we may assume $a_{1 i}=0$ for all $i$. If $a_{2 i}=0$ for some $i$, then the base-change theorem gives $E \cong \pi_{i}^{*}(F)$ for some weakly uniform vector bundle $F$ on $X_{i}$. If $s=3$, then we are done. In the general case we reduce to the case $s^{\prime}:=s-1$. Thus, to complete the proof it is sufficient either to obtain a contradiction or to get that $E$ splits under the additional condition that $a_{2 i}<0$ for all $i$ and $s \geq 3$. Applying the base-change theorem to $\pi_{1 *}$ we get that $E$ fits in the following extension:

$$
0 \rightarrow \mathcal{O}\left(0, c_{2}, \ldots, c_{s}\right) \rightarrow E \rightarrow \mathcal{O}\left(a_{1,2}, d_{2}, \ldots, d_{s}\right) \rightarrow 0 .
$$

Since $-a_{1,2} \geq 0$, Künneth's formula shows that (2.2) splits unless $n_{i}=1$ for all $i \geq 2$. Using $\pi_{2 *}$ instead of $\pi_{1 *}$ we get that $E$ splits unless $n_{1}=1$.

\section{Higher rank weakly uniform vector bundles}

Now we consider higher rank weakly uniform vector bundles.

PRoposition 3.1. Let $E$ be a rank $r$ weakly uniform vector bundle on $X$ with splitting type $(0, \ldots, 0)$. Then $E$ is trivial.

Proof. The case $s=1$ is true by [8, Theorem 3.2.1]. Hence we may assume $s \geq 2$ and use induction on $s$. By the inductive assumption, $E \mid \pi_{1}^{-1}(P)$ is trivial for each $P \in \mathbb{P}^{n_{1}}$. By the base-change theorem, $F:=\pi_{1 *}(E)$ is a rank $r$ vector bundle on $X_{1}$ and the natural map $\pi_{1}^{*}(F) \rightarrow E$ is an isomorphism. This isomorphism implies that $F$ is uniform of splitting type $(0, \ldots, 0)$. Hence, the inductive assumption gives that $F$ is trivial. Thus $E$ is trivial.

In order to study uniform vector bundles with $a_{1}>\cdots>a_{r}$ we need the following lemmas.

LEMMA 3.2. Fix an integer $r \geq 2$ and a rank $r$ vector bundle on $X$. Assume the existence of an integer $i \in\{1, \ldots, s\}$ such that $E \mid \pi_{i}^{-1}(P)$ is the direct sum of line bundles for all $P \in X_{i}$. If $n_{i}=1$ assume that the splitting type of $E \mid \pi_{i}^{-1}(P)$ is the same for all $P \in X_{i}$. Let $\left(a_{1}, \ldots, a_{r}\right)=\left(b_{1}^{m_{1}}, \ldots, b_{k}^{m_{k}}\right), b_{1}>\cdots>b_{k}, m_{1}+\cdots+$ $m_{k}=r$, be the splitting type of $E \mid \pi^{-1}(P)$ for any $P \in X_{i}$. Then there are $k$ vector 
bundles $F_{1}, \ldots, F_{k}$ on $X_{i}$ and $k$ vector bundles $E_{1}, \ldots, E_{k}$ on $X$ such that $\operatorname{rank}\left(F_{i}\right)=m_{i}, E_{k}=E, E_{i-1}$ is a subbundle of $E_{i}$ and $E_{i} / E_{i-1} \cong \pi_{i}^{*}\left(F_{i}\right)\left(-b_{i}\right)$ (with the convention $E_{0}=0$ ).

PROOF. Notice that even in the case $n_{i} \geq 2$ the splitting type of $E \mid \pi^{-1}(P)$ does not depend on the choice of $P \in X_{i}$ (for example, use Chern classes or local rigidity of direct sums of line bundles). Thus, $E \mid \pi_{i}^{-1}(P) \cong \bigoplus_{j=1}^{k} \mathcal{O}_{\pi_{i}^{-1}(P)}\left(b_{j}\right)^{\oplus m_{j}}$ for all $P \in X_{i}$.

Set $F_{1}:=\pi_{i *}\left(E\left(0, \ldots,-b_{1}, \ldots, 0\right)\right)$. By the base-change theorem, $F_{1}$ is a rank $m_{1}$ vector bundle on $X_{i}$ and the natural map $\rho: \pi_{i}^{*}\left(F_{1}\right)\left(0, \ldots, b_{1}, \ldots\right) \rightarrow E$ is a vector bundle embedding, that is, either $\rho$ is an isomorphism (case $r=m_{1}$ ) or $\operatorname{Coker}(\rho)$ is a rank $r-m_{1}$ vector bundle on $X$. If $m_{1}=r$, then $k=1$ and we are done. Now assume $k \geq 2$, that is, $m_{1}<r$. Fix any $P \in X_{i}$. By definition, $\operatorname{Coker}(\rho)$ fits in an exact sequence of vector bundles on $X$ :

$$
0 \rightarrow \pi_{i}^{*}\left(F_{1}\right)\left(0, \ldots, b_{1}, \ldots, 0\right) \rightarrow E \rightarrow \operatorname{Coker}(\rho) \rightarrow 0
$$

and the restriction to $\pi_{i}^{-1}(P)$ of the injective map of (3.1) induces an embedding of vector bundles $j_{P}: \mathcal{O}_{\pi_{i}^{-1}(P)}\left(b_{1}\right)^{\oplus m_{1}} \rightarrow \bigoplus_{j=1}^{k} \mathcal{O}_{\pi_{i}^{-1}(P)}\left(b_{j}\right)^{\oplus m_{j}}$. Since $b_{1}>b_{j}$ for all $j>1$, we get

$$
\operatorname{Coker}\left(j_{P}\right) \cong \bigoplus_{j=2}^{k} \mathcal{O}_{\pi_{i}^{-1}(P)}\left(b_{j}\right)^{\oplus m_{j}}
$$

We apply to $\operatorname{Coker}(\rho)$ the inductive assumption on $k$.

LEMMA 3.3. Assume $s=2$ and $n_{1} \geq 2, n_{2} \geq 3$. Fix an integer $r$ such that $3 \leq r \leq n_{2}$ and $a$ rank $r$ uniform vector bundle $E$ with splitting type $a_{1}>\cdots>a_{r}$. Then $E$ is isomorphic to a direct sum of $r$ line bundles.

PROOF. Since $r \geq 3$, we have $a_{r} \leq a_{1}-2$. Thus, the classification of uniform vector bundles on $\mathbb{P}^{n_{2}}$ with rank $r \leq n_{2}$ gives $E \mid \pi_{1}^{-1}(P) \cong \bigoplus_{i=1}^{r} \mathcal{O}_{\pi_{1}^{-1}(P)}\left(a_{i}\right)$ for all $P \in \mathbb{P}^{n_{1}}$. Apply Lemma 3.2 with respect to the integers $i=1$ and $k=r$ and let $F_{i}, E_{i}, 1 \leq i \leq r$, be the vector bundles given by the lemma. Since $E_{r}=E$, it is sufficient to prove that each $E_{i}$ is a direct sum of $i$ line bundles. Since $\operatorname{rank}\left(E_{i}\right)=i$, the latter assertion is obvious if $i=1$. Fix an integer $i$ such that $1 \leq i<r$ and assume that $E_{i}$ is isomorphic to a direct sum of $i$ line bundles. Lemma 3.2 gives an extension

$$
0 \rightarrow E_{i} \rightarrow E_{i+1} \rightarrow L \rightarrow 0
$$

with $L$ a line bundle on $\mathbb{P}^{n_{1}} \times \mathbb{P}^{n_{2}}$. Since $n_{1} \geq 2$ and $n_{2} \geq 2$, Künneth's formula gives that any extension of two line bundles on $\mathbb{P}^{n_{1}} \times \mathbb{P}^{n_{2}}$ splits. Thus $E_{i+1}$ is a direct sum of $i+1$ line bundles. 
PROPOSITION 3.4. Fix an integer $r \geq 3$ and a rank $r$ uniform vector bundle on $X$ with splitting type $a_{1}>\cdots>a_{r}$. Assume $s \geq 2, n_{2} \geq r$ and $n_{i} \geq 2$ for all $i \neq 2$. Then $E$ is isomorphic to a direct sum of $r$ line bundles.

Proof. The case $s=2$ is Lemma 3.3. Thus we may assume $s \geq 3$ and that the proposition is true for $\mathbb{P}^{n_{1}} \times \cdots \times \mathbb{P}^{n_{s-1}}$. By the inductive assumption, $E \mid u_{s}^{-1}(P) \cong$ $\bigoplus_{i=1}^{r} \mathcal{O}_{u_{s}^{-1}(P)}\left(a_{i}, \ldots, a_{i}\right)$ for all $P \in \mathbb{P}^{n_{s}}$. As in the proof of Lemma 3.2, taking instead of $\pi_{i}$ the projection $u_{i}: X \rightarrow \mathbb{P}^{n_{i}}$, we get line bundles $L_{i}, 1 \leq i \leq r$, of $\mathbb{P}^{n_{s}}$ (that is, line bundles $u_{i}^{*}(L) \cong \mathcal{O}\left(0, \ldots, 0, c_{i}, 0, \ldots, 0\right)$ on $\left.X\right)$ and subbundles $E_{1} \subset$ $E_{2} \subset \cdots E_{r}=E$ such that $E_{i} / E_{i-1} \cong \mathcal{O}_{X}\left(a_{i-1}, \ldots, a_{i-1}, c_{i}\right)$ (with the convention $\left.E_{0}=0\right)$. It is sufficient to prove that each $E_{i}$ is isomorphic to a direct sum of $i$ line bundles. Since this is obvious for $i=1$, we may use induction on $i$. Fix an integer $i \in\{2, \ldots, r\}$. Our assumption on $X$ implies that the extension of any two line bundles splits. Hence, $E_{i} \cong E_{i-1} \oplus \mathcal{O}_{X}\left(a_{i-1}, \ldots, a_{i-1}, c_{i}\right)$.

\section{References}

[1] E. Ballico and P. Ellia, 'Fibrés uniformes de rang 5 sur $\mathbb{P}^{3}$ ', Bull. Soc. Math. France 111 (1983), 59-87.

[2] E. Ballico and P. E. Newstead, 'Uniform bundles on quadric surfaces and some related varieties', J. Lond. Math. Soc. (2) 31(2) (1985), 211-223.

[3] J. M. Drezet, 'Exemples de fibres uniformes nonhomogènes $\operatorname{sur} \mathbf{P}_{n}{ }^{\prime}, C$. R. Acad. Sci. Paris Sér. A 291 (1980), 125-128.

[4] G. Elencwajg, 'Les fibrés uniformes de rang 3 sur $\mathbf{P}_{2}(\mathbf{C})$ sont homegènes', Math. Ann. 231 (1978), 217-227.

[5] G. Elencwajg, A. Hirschowitz and M. Schneider, 'Les fibres uniformes de rang au plus $n$ sur $\mathbf{P}_{n}(\mathbf{C})$ sont ceux qu'on croit', in: Vector Bundles and Differential Equations (Proc. Conf., Nice, 1979), Progress in Mathematics, 7 (Birkhäuser, Boston, MA, 1980), pp. 37-63.

[6] P. Ellia, 'Sur les fibrés uniformes de rang $(n+1)$ sur $\mathbb{P}^{n}$ ', Mém. Soc. Math. Fr. 7 (1982), 59-87.

[7] P. E. Newstead and R. L. E. Schwarzenberger, 'Reducible vector bundles on a quadric surface', Proc. Cambridge Philos. Soc. 60 (1964), 421-424.

[8] Ch. Okonek, M. Schneider and H. Spindler, Vector Bundles on Complex Projective Spaces, Progress in Mathematics, 3 (Birkhäuser, Boston, MA, 1980).

[9] E. Sato, 'Uniform vector bundles on a projective space', J. Math. Soc. Japan 28 (1976), 123-132.

[10] R. L. E. Schwarzenberger, 'Reducible vector bundles on a quadric surface', Proc. Cambridge Philos. Soc. 58 (1962), 209-216.

[11] A. Van de Ven, 'On uniform vector bundles', Math. Ann. 195 (1972), 245-248.

EDOARDO BALLICO, Università di Trento, 38123 Povo (TN), Italy e-mail: ballico@science.unitn.it

FRANCESCO MALASPINA, Politecnico di Torino, Corso Duca degli Abruzzi 24, 10129 Torino, Italy

e-mail: francesco.malaspina@polito.it 